\title{
OPTIMIZING PHASE COMPRESSION FOR TRANSIT SIGNAL PRIORITY AT ISOLATED INTERSECTIONS
}

\author{
Xuedong Hua ${ }^{1}$, Wei Wang ${ }^{2}$, Yinhai Wang ${ }^{3}$, Ziyuan $\mathrm{Pu}^{4}$ \\ ${ }^{1,2}$ Jiangsu Key Laboratory of Urban ITS, Southeast University, Nanjing, China \\ ${ }^{3,4}$ Dept of Civil and Environmental Engineering, University of Washington, Seattle, United States
}

Submitted 5 April 2015; resubmitted 30 September 2015; accepted 31 December 2015

\begin{abstract}
Transit signal priority (TSP) is a promising low-cost strategy that gives preferential treatments for the buses to go through intersections with minimum delay time. In this paper, a new TSP control model was presented for isolated intersections to minimize bus delay and to reduce the impact of TSP on other vehicles by optimizing signal control phase selection and compression. This paper starts with the phase selection and compression strategies to provide treatments to bus priority requests. Then, two new features on phase selection and compression aspects are applied to TSP, i.e. the time that a bus priority request needs is provided by the phase(s) with the lowest traffic volume, and multi-phases can be selected to serve a bus request. Field data are collected from a major traffic corridor in Changzhou (China) and applied for VISSIM simulation. The proposed TSP control model as well as the fixed-time control and the conventional TSP control models are tested and compared under different traffic demands, headways and maximum saturation degrees. The comparative results showed that the proposed model outperformed the conventional TSP control model in terms of reducing bus delay, minimizing the impact on other vehicles and reducing the stop rate for buses. This paper reveals that, the proposed TSP strategy can significantly optimize the phase compression process and improve transit efficiency.
\end{abstract}

Keywords: transit signal priority; phase selection and compression; optimization; multi-phases compression; VISSIM simulation.

\section{Introduction}

To address the increasing traffic congestion in urban areas, both researchers and government officers are seeking for effective yet inexpensive solutions that do not involve new infrastructure construction (or with few infrastructure construction) to enhance transportation system performance. A good way to achieve this is to give signal priority to transit vehicles at intersections. Transit Signal Priority (TSP) is a promising low-cost strategy that can enhance transit operations at signalized intersections. With TSP, buses can communicate with signal controllers to claim right of way and pass through intersections with lower or even no delay. Proper TSP strategies can significantly reduce bus delay and improve average bus speed, and thus increasing attractiveness of transit system to the public. In addition, the decrease of wait time at intersections by TSP can reduce bus headway fluctuations, which improves the reliability of buses (Li, Zhang 2012). Therefore, after the earliest bus preemption experiment conducted in the US (US Department of Housing... 1968a, 1968b, 1968c), TSP strategies have been imple- mented widely in metropolitan areas, such as Seattle, Portland, Los Angeles, and Chicago (Liao, Davis 2007).

The first TSP study can date back to 1979, where the advantages and weaknesses were discussed (Salter, Shahi 1979). Since then, a handful of early TSP related studies, in which the basic of concepts and principles of TSP emerges, are conducted (Jacobson, Sheffi 1981; Chang, Messer 1985; Khasnabis et al. 1991; Al-Sahili, Taylor 1996; Garrow, Machemehl 1997). In 2005, the planning and implementation handbook for TSP summarized the three key components of TSP system: transit vehicle detection, prediction algorithm of arrival time, and TSP control strategy (Smith et al. 2005). Although a handful of researchers focused on bus detection and arrival time prediction (Lee et al. 2005; Kim, Rilett 2005; Tan et al. 2008; Ekeila et al. 2009), TSP control strategy development as well as implementation is the major research issue. The related studies mainly concentrate on several representative topics:

- TSP strategies logic development for an isolated intersection (Balke et al. 2000; Dion, Hellinga 
2002; Polgár et al. 2013; Ahmed 2014; Wang et al. 2014; Hu et al. 2014; Wolput et al. 2015);

- TSP strategies optimization for coordinated intersections and stops (Duerr 2000; Janos, Furth 2002; Wahlstedt 2011; Delgado et al. 2015);

- high-frequency multiple (or conflicting) bus requests issues (Christofa, Skabardonis 2011; Ma et al. 2013; Zeng et al. 2014);

- conditional TSP implementation to buses behind schedule (Lin 2002; Li and Zhang 2012; Hu et al. 2015).

All these TSP strategy studies can be classified into three major categories: passive priority, active priority, and TSP operating in real-time. Passive priority requires no specialized hardware and typically involves optimizing signal timing based on knowledge of transit route and ridership patterns to create a green wave for transit vehicles (Ma, Yang 2007). Active TSP techniques rely on detecting transit vehicles arrival time at an intersection and adjusting the signal timing dynamically to reduce the bus delay (Janos, Furth 2002; Wahlstedt 2011; Li, Zhang 2012; Ma et al. 2013; Ahmed 2014; Wang et al. 2014; Zeng et al. 2014; Wolput et al. 2015).TSP operating in real-time shares the same TSP strategies such as early green, green extension and phase insertion with active priority, and provides priority while simultaneously optimizing traffic performance (Balke et al. 2000; Duerr 2000; Zhou, Gan 2009; Christofa, Skabardonis 2011; Hu et al. 2014, 2015).

Based on the review of literature on TSP strategies, two shortcomings were identified. First, while previous studies addressed critical issues on the optimal TSP strategies, most of them were based on the widely applied phase selection and compression method. That is, the phase in which a bus arrives with priority request will be selected and compressed to provide time for the request (Balke et al. 2000; Ma, Yang 2007; Li, Zhang 2012; Ahmed 2014; Wang et al. 2014; Hu et al. 2014, 2015). However, it is not an optimal method. Especially when the traffic volume in the bus arrival phase is relatively high, the compression of the phase can result in a larger delay increase for other vehicles. Second, although some new research ideas are observed such as compressing all phases to provide time for a TSP request (Lin 2002; Zhou, Gan 2009; Li et al. 2012), the common practice is to compress a single phase (at most two phases if phase insertion strategy is used). In fact, when one phase cannot afford enough time for a bus request, compressing multi-phases can provide more time and decrease the bus delay. However, no attempt was found in our literature review to find the optimal number of compressed phases. This paper addresses the following two research questions:

- is the widely accepted method that compresses the bus arrival phase to provide time for a TSP treatment optimal?

- which strategies perform the best to provide time for a TSP request: compressing a single phase, compressing some specifically selected phases, or compressing all possible phases?
The remainder of the paper is structured as follows. First, the problem about how to select and compress phases for a bus request is posed and discussed in Section 1. Next in Section 2, a new TSP control model is formulated, in which the time a TSP request needs is provided by phase(s) with the lowest traffic volume, and by multi-phases if one phase cannot afford the time needed. Section 3 describes the data collection information and VISSIM model setup process. Simulation results of the proposed TSP model and the analysis are shown in Section 4. Conclusions and limitations are given in the last section.

\section{Problem Statement}

If the cycle length remains constant, providing time for a TSP request indicates that a phase green time is compressed. In previous studies, a TSP request is generally served by compressing the bus arrival phase, no matter which TSP strategy is used. However, except for compressing the bus arrival phase, some other alternatives may also be available, such as compressing a rear phase or all possible phases. In fact, how to select and compress phases for a TSP request is very important not only for buses to acquire right of way, but also for other vehicles to relieve the impact of TSP implementation. To the best of our knowledge, no previous studies addressed this issue.

Fig. 1 demonstrates two examples when a bus arrives at a non-transit phase. In the figure, Phase 1 is the transit phase during which transit vehicles have right of way, and the base TSP strategy is the commonly applied method to provide time for a TSP request. In Fig. 1a, when Phase 2 can afford the green extension time by itself, usually it will be compressed (base TSP strategy). What if Phase 3 (Strategy A) or 4 (Strategy B) can be compressed to serve the TSP request as well?
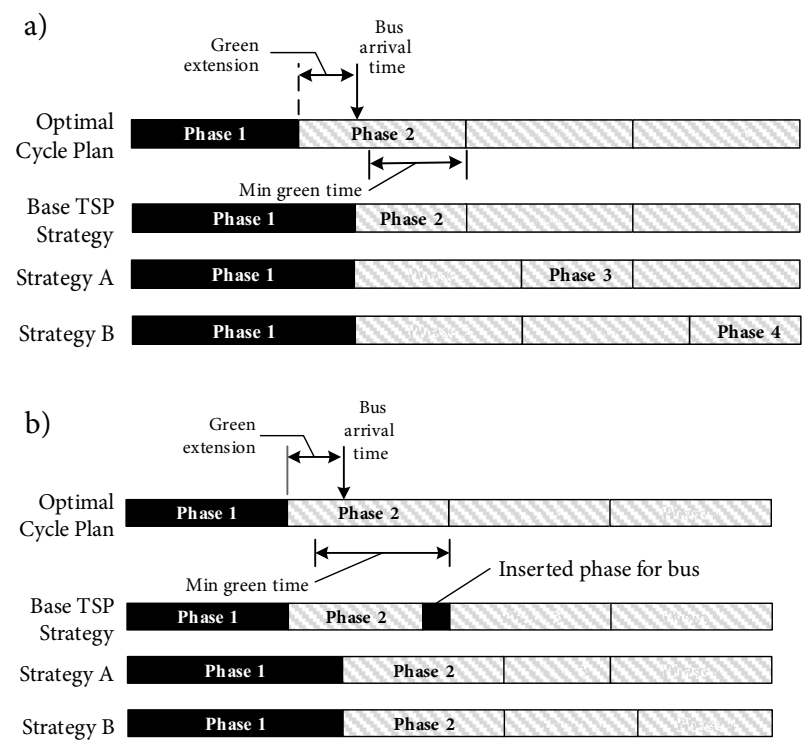

Fig. 1. Signal timing plans under different TSP strategies: a - compressible time of Phase 2 is larger than the green extension time; $b$ - compressible time of Phase 2 is smaller than the green extension time 
Although all these strategies are same for the bus to unimpeded pass through the intersection, they can result in different impacts on other vehicles. Given that the traffic volume for Phase 2 is relatively high while traffic volumes for Phases 3 and 4 are low, the Strategies $\mathrm{A}$ and $\mathrm{B}$ can cause lower delay for other vehicles when compared with the base TSP strategy. In Fig. 1b, when Phase 2 cannot provide enough time for green extension, the bus has to stop to wait for the next priority window (base TSP strategy). Under this circumstance, if Phase 3 can afford the green extension time, Strategy $\mathrm{A}$ in Fig. $1 \mathrm{~b}$ can be adopted. Even if no single phase can provide enough time for TSP request, multi-phases can serve the bus request together, such as compressing Phases 3 and 4 (Strategy B). Compared with base TSP strategy, Strategies A and B in Fig. $1 \mathrm{~b}$ can significantly reduce the bus delay.

In this paper, two changes are made to optimize phase selection and compression strategy. First, the time that a TSP request needs is not provided by the bus arrival phase any more, but by the phase(s) with the lowest traffic volume; Second, multi-phases can be selected to serve the TSP request together if one phase cannot afford the time needed. Compressing the phase with the lowest traffic volume can relieve the impact of TSP strategies on other vehicles, while multi-phases compression can reduce the average bus delay. As a result, both buses and other vehicles can benefit from the above changes.

\section{Modelling}

The primary objective of the proposed TSP model is to minimize bus delay and to reduce the impact on other vehicles by optimizing signal control phase selection and compression. It contains four main steps:

- calculate the green time adjustment constraints of each phase;

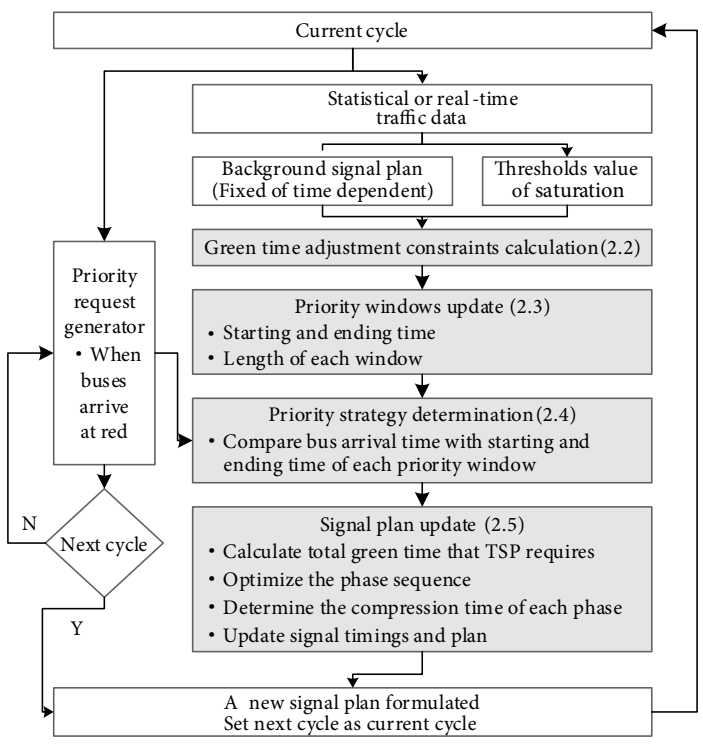

Fig. 2. the Framework of the proposed TSP model
- update the status of priority windows (priority window is a time constraint for TSP provision: when a bus arrives at an intersection within a priority window, the bus can be served by TSP and pass through the intersection immediately without a stop; otherwise, it has no choice but to stop and wait for the following priority window);

- determine the adopted priority strategies;

- update the signal plan under TSP requests.

The interrelation among these steps as well as their principal components is illustrated in Fig. 2. Some more detailed information of each step is described in this section.

\subsection{Notations}

To make the model presentation more facilitative, the major parameters are summarized first in Table 1. The set of decision variables are specified as shown in Table 2 .

Table 1. Summary of parameters

\begin{tabular}{|c|c|}
\hline Notions & Meaning \\
\hline$g_{i, j}$ & length of green time of phase $i$ in cycle $j[\mathrm{~s}]$ \\
\hline$s_{i}$ & saturation flow rate of phase $i[\mathrm{veh} / \mathrm{h}]$ \\
\hline$q_{i}$ & $\begin{array}{l}\text { traffic flow rate of critical movement of phase } i \\
\text { [veh/h] }\end{array}$ \\
\hline$C_{j}$ & length of cycle $j$ [s] \\
\hline$g_{i, j}^{\min }$ & minimum green time of phase $i$ in cycle $j[\mathrm{~s}]$ \\
\hline$x_{i}^{\max }$ & allowable maximum saturation degree of phase $i$ \\
\hline$k_{i, j}^{\max }$ & $\begin{array}{l}\text { maximum compressible time of phase } i \text { in cycle } \\
j[\mathrm{~s}]\end{array}$ \\
\hline$I$ & inter-green time between two phases [s] \\
\hline$g_{i, j}^{S}$ & initial green starting time of phase $i$ in cycle $j$ [s] \\
\hline$g_{i, j}^{E}$ & initial green ending time of phase $i$ in cycle $j[\mathrm{~s}]$ \\
\hline$t t$ & $\begin{array}{l}\text { average time for buses to get through the } \\
\text { intersection }[\mathrm{s}]\end{array}$ \\
\hline$P_{i, j}^{S}$ & starting time of priority windows $i$ in cycle $j[\mathrm{~s}]$ \\
\hline$P_{i, j}^{E}$ & ending time of priority windows $i$ in cycle $j[\mathrm{~s}]$ \\
\hline$L_{i, j}$ & length of priority windows $i$ in cycle $j[\mathrm{~s}]$ \\
\hline$N$ & number of phases \\
\hline$b_{j}^{a}$ & bus arrival time at the at cycle $j[\mathrm{~s}]$ \\
\hline$C_{j}^{L}$ & $\begin{array}{l}\text { total compression time from Phase } 1 \text { to phase } m \\
\text { in cycle } j[\mathrm{~s}]\end{array}$ \\
\hline$C_{j}^{R}$ & $\begin{array}{l}\text { total compression time from phase } m+1 \\
\text { to phase } N \text { in cycle } j[\mathrm{~s}]\end{array}$ \\
\hline$C_{i, j}$ & compression time of phase $i$ in cycle $j[\mathrm{~s}]$ \\
\hline
\end{tabular}


Table 2. Summary of decision variables

\begin{tabular}{|c|l|}
\hline Notions & \multicolumn{1}{|c|}{ Meaning } \\
\hline$P_{j}$ & $\begin{array}{l}\text { actual priority strategy which is selected for } \\
\text { priority request in cycle } j\end{array}$ \\
\hline$m$ & $\begin{array}{l}\text { the priority window No. in which the priority } \\
\text { request are served }\end{array}$ \\
\hline$g_{i, j}^{E^{*}}$ & $\begin{array}{l}\text { green ending time of phase } i \text { in cycle } j \text { after TSP } \\
\text { strategy applied [s] }\end{array}$ \\
\hline$g_{i, j}^{S^{*}}$ & $\begin{array}{l}\text { green starting time of phase } i \text { in cycle } j \text { after TSP } \\
\text { strategy applied [s] }\end{array}$ \\
\hline$g_{i n s, j}^{S}$ & $\begin{array}{l}\text { green starting time of the inserted phase for } \\
\text { priority request in cycle } j \text { [s] }\end{array}$ \\
\hline$g_{i n s, j}^{S}$ & $\begin{array}{l}\text { green ending time of the inserted phase for } \\
\text { priority request in cycle } j \text { [s] }\end{array}$ \\
\hline
\end{tabular}

\subsection{Minimum Green Time and Maximum Compressible Time Calculation}

The minimum green time is updated as shown in Eq. (1):

$$
g_{i, j}^{\min }=\frac{C_{j} \cdot q_{i}}{s_{i} \cdot x_{i}^{\max }} .
$$

The maximum compressible green time is then achieved by Eq. (2):

$$
k_{i, j}^{\max }=g_{i, j}-g_{i, j}^{\min } .
$$

\subsection{Priority Windows Update}

The starting and ending time of priority windows $i$ are updated by Eqs (3) and (4):

$$
\begin{aligned}
& P_{i, j}^{S}=\left\{\begin{array}{l}
g_{i, j}^{E}, \text { if } i=1 ; \\
g_{i, j}^{E}-\sum_{t=1}^{i} k_{t, j}^{\max }+I, \text { otherwise. }
\end{array}\right. \\
& P_{i, j}^{E}=\left\{\begin{array}{l}
g_{i+1, j}^{S}+\sum_{t=i+1}^{N} k_{t, j}^{\max }-I-t t, \text { if } i<N ; \\
g_{i, j}^{E}-t t-I, \text { otherwise. }
\end{array}\right.
\end{aligned}
$$

In Eqs (3) and (4), the sum of maximum compressible time indicates that in the proposed TSP strategy, the time a TSP request needs can be provided by multiphases.

By calculating starting time and ending time of priority windows, we obtain the length of each priority window, as shown in Eq. (5):

$$
L_{i, j}=P_{i, j}^{E}-P_{i, j}^{S}=\left\{\begin{array}{l}
\sum_{t=2}^{N} k_{t, j}^{\max }-t t, \text { if } i=1 ; \\
\sum_{t=1}^{N} k_{t, j}^{\max }-t t-I, \text { if } N>i>1 ; \\
\sum_{t=1}^{N} k_{t, j}^{\max }-t t, \text { if } i=N .
\end{array}\right.
$$

\subsection{Priority Strategies Determination}

Given the predicted bus arrival time at stop line, we determine the actual priority strategy for a TSP request in Eq. (6):

$$
P_{j}=\left\{\begin{array}{l}
1, \text { if } P_{1, j}^{E} \geq b_{j}^{a} \geq P_{1, j}^{S} \\
2, \text { if } P_{N, j}^{E} \geq b_{j}^{a}>P_{N-1, j}^{E} ; \\
3, \text { if } P_{N-1, j}^{E} \geq b_{j}^{a}>P_{1, j}^{E} .
\end{array}\right.
$$

In Eq. (6), $P_{j}=1$ means that green extension strategy is employed; $P_{j}=2$ means red truncation strategy is selected; And $P_{j}=3$ indicates that phase insertion strategy is selected. Note that, when red truncation or phase insertion strategy is used (i.e. $P_{j}=2$ or 3 ), there are two situations according to the relationship between the bus arrival time and priority windows:

- if the bus arrives exactly within a priority window, it can pass through the intersection without a stop;

- if the arrival time is slightly ahead of the starting time of a priority window, the bus needs to stop and wait for the starting time.

Adding these situations into Eq. (6), the actual priority strategy is further extended as in Eq. (7):

$$
P_{j}=\left\{\begin{array}{l}
1, \text { if } P_{m, j}^{E} \geq b_{j}^{a} \geq P_{m, j}^{S} \text { and } m=1 ; \\
21, \text { if } P_{m, j}^{E} \geq b_{j}^{a} \geq P_{m, j}^{S} \text { and } m=N ; \\
22, \text { if } P_{m, j}^{S}>b_{j}^{a}>P_{m-1, j}^{E} \text { and } m=N ; \\
31, \text { if } P_{m, j}^{E} \geq b_{j}^{a} \geq P_{m, j}^{S} \text { and } N>m>1 ; \\
32, \text { if } P_{m, j}^{S} \geq b_{j}^{a} \geq P_{m-1, j}^{E} \text { and } N>m>1 .
\end{array}\right.
$$

In Eq. (7), $m$ denotes the priority window in which the arrival bus will be served. Similar to Eq. (6), when $P_{j}=2 \cdot A$ ( $A$ is equal to 1 or 2 ), red truncation strategy is selected, while $P_{j}=3 \cdot A$, phase insertion strategy is employed. The second number, namely $A$, denotes the two situations. When $A$ is equal to 1 , the bus can be served with a certain TSP strategy and pass through the intersection without a stop, while $A$ is equal to 2 , the bus needs to stop and wait for the green time.

\subsection{Green Starting and Ending Time Update}

When a TSP request is served in priority window $m$, the ending time of phase $m$ and the staring time of phase $m+1$ (when $m$ is equal to $N, m+1$ phase indicates the first phase of the next cycle) are determined by Eqs (8) and (9):

$$
g_{m, j}^{E^{*}}=\left\{\begin{array}{l}
b_{j}^{a}, \text { if } P_{j}=1 ; \\
g_{m, j}^{E}-\sum_{t=1}^{m} k_{t, j}^{\max }, \text { if } P_{j}=22 \text { or } 32 \\
\min \left(g_{m, j}^{E}, b_{j}^{a}-I\right), \text { if } P_{j}=21 \text { or } 31 .
\end{array}\right.
$$




$$
g_{m+1, j}^{S^{*}}=\left\{\begin{array}{l}
b_{j}^{a}+t t+I, \text { if } P_{j}=1 ; \\
\max \left(g_{1, j+1}^{S}, g_{m, j}^{E^{*}}+t t+I\right), \text { if } P_{j}=21 \text { or } 22 ; \\
\max \left(g_{m+1, j}^{S}, g_{m, j}^{E^{*}}+t t+2 \cdot I\right), \text { if } P_{j}=31 \text { or } 32 .
\end{array}\right.
$$

In the proposed model, the green time that a TSP request needs is provided by phases with the lowest traffic volume. Therefore, the green starting and ending time of all the phases should be carefully checked and updated.

Given the ending time of phase $m$ and the staring time of phase $m+1$, we obtain the total compression time of phases from 1 to $m$, and from $m+1$ to $N$ as shown in Eqs (10) and (11):

$$
\begin{aligned}
C_{j}^{L} & =\left\{\begin{array}{l}
0, \text { if } m=1 ; \\
g_{m, j}^{E}-g_{m, j}^{E^{*}}, \text { if } m>1 ;
\end{array}\right. \\
C_{j}^{R} & =\left\{\begin{array}{l}
g_{m+1, j}^{S^{*}}-g_{m+1, j}^{S} \\
0, \text { if } m=N .
\end{array} \text { if } m<N ;\right.
\end{aligned}
$$

Given the total compression times, we can then assign the total compression time to each phase and update the starting and ending time.

First, phases from 1 to $m$, and from $m+1$ to $N$ are sorted in order of volume (low to high), and denoted as $\left(L_{1}, L_{2}, \ldots, L_{m}\right)$ and $\left(R_{m+1}, R_{m+2}, \ldots, R_{N}\right)$, separately. Then, we can assign the total compression time with the First Come First Compression (FCFC) rule: the first phase of each sequence will be compressed first; when the first phase cannot be compressed any more, it would be removed from the sequence and the following phase in the sequence will be then sequentially compressed. By the FCFC rule, the compression time of phases from 1 to $m$ are calculated iteratively by Eq. (12):

$$
\begin{aligned}
& C_{L_{1}, j}=\min \left(k_{L_{1}, j}^{\max }, C_{j}^{L}\right) ; \\
& C_{L_{2}, j}=\min \left(k_{L_{2}, j}^{\max }, C_{j}^{L}-C_{L_{1}, j}\right) ; \\
& \vdots \\
& C_{L_{m}, j}=\min \left(k_{L_{m}, j}^{\max }, C_{j}^{L}-\sum_{t=1}^{m-1} C_{L_{t}, j}\right) .
\end{aligned}
$$

Similarly, the compression time of phases from $m+1$ to $N$ are obtained by Eq. (13):

$$
\begin{aligned}
& C_{R_{m+1}, j}=\min \left(k_{R_{m+1}, j}^{\max }, C_{j}^{R}\right) ; \\
& C_{R_{m+2}, j}=\min \left(k_{L_{m+2}, j}^{\max }, C_{j}^{R}-C_{R_{m+1}, j}\right) ; \\
& \vdots \\
& C_{R_{N}, j}=\min \left(k_{L_{N}, j}^{\max }, C_{j}^{R}-\sum_{t=m+1}^{N-1} C_{L_{t}, j}\right) .
\end{aligned}
$$

By Eqs (12) and (13), all the compression time of each phase are obtained. Since the ending time of phase $m$ and the staring time of phase $m+1$ have already been determined by Eqs (8) and (9), the starting and ending time of phases before $g_{m, j}^{E^{*}}$ and after $g_{m+1, j}^{S^{*}}$ are calculated respectively, as shown in Eqs (14) and (15):

$$
\begin{aligned}
& g_{i, j}^{E^{*}}=g_{i, j}^{S^{*}}+g_{i, j}-C_{i, j} ; \\
& g_{i+1, j}^{S^{*}}=g_{i, j}^{E^{*}}+I .
\end{aligned}
$$

In Eqs (14) and (15), the calculation needs an initial value $g_{1, j}^{S^{*}}$ to start with. If $g_{1, j}^{S^{*}}$ has not been updated by Eq. (9), the value of $g_{1, j}^{S^{*}}$ should be set to the initial starting time $g_{1, j}^{\mathrm{S}}$.

Note that, when $N>m>1$, phase insertion strategy is employed, it indicates that there will be one more 'phase' (i.e. the inserted phase). The green starting and ending time of the inserted phase should be updated as well by Eqs (16) and (17):

$$
\begin{aligned}
& g_{i n s, j}^{S}=g_{m, j}^{E^{*}}+I ; \\
& g_{i n s, j}^{E}=g_{\text {ins }, j}^{S}+t t .
\end{aligned}
$$

Although the newly proposed TSP strategy seems a little more complicated, which contains lots of equations and parameters, the implementation of the new strategy and the measurement work of parameters are as easy as those in previous TSP strategies. More detailed information about the implementation issues are illustrated in Appendix.

\section{Data Collection and VISSIM Model Setup}

To verify the performance of the proposed TSP control strategy under different traffic conditions, simulation method was applied. VISSIM, one of the most widely used transportation research tool, was adopted for Simulation in this section. The simulation environment was carefully calibrated with the help of previous studies and some field data collected from Changzhou (China).

\subsection{Field Data Collection}

A real-world intersection of Tongjiang Avenue and Taihu Avenue from Changzhou was selected. Tongjiang Avenue is a major corridor in Changzhou with exclusive bus lanes in both approaches. Along Tongjiang Avenue, there is a major Bus Rapid Transit (BRT) route. Fig. 3 illustrates the layout of the test intersection as well as the optimal cycle plan.

A video camera was set up in the field to collect the traffic data. The camera was placed on top of a shopping mall to achieve an adequate viewing height to cover the whole intersection. Data collection was conducted during a weekday (28 June 2012), from 4:00 PM to 5:30 PM. The traffic volume data of each approach were collected to calculate the peak hour traffic flow rate; and to calibrate the VISSIM model. Table 3 shows the traffic flow rate for simulation. 


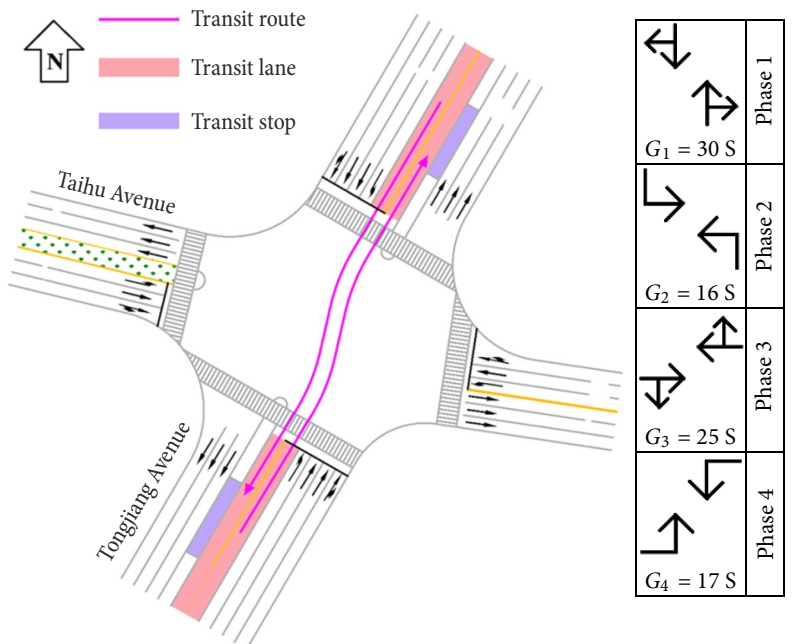

Fig. 3. Phase design and layout of the test intersection

Table 3. Basic traffic flow rate

\begin{tabular}{|l|c|c|}
\hline Intersection approach & $\begin{array}{c}\text { Movement } \\
\text { direction }\end{array}$ & $\begin{array}{c}\text { Flow rate } \\
\text { [veh/h] }\end{array}$ \\
\hline \multirow{3}{*}{ Eastbound (EB) } & left & 210 \\
\cline { 2 - 3 } & through & 166 \\
\cline { 2 - 3 } Northbound (NB) & right & 144 \\
\hline & left & 200 \\
\hline \multirow{3}{*}{ Westbound (WB) } & through & 1010 \\
\cline { 2 - 3 } & right & 40 \\
\cline { 2 - 3 } & left & 105 \\
\hline \multirow{3}{*}{ Southbound (SB) } & through & 310 \\
\hline & right & 45 \\
\cline { 2 - 3 } & left & 202 \\
\cline { 2 - 3 } & through & 1140 \\
\hline
\end{tabular}

\subsection{VISSIM Simulation Setup}

VISSIM (Version 5.40), the widely applied microcosmic simulation tool, was adopted to evaluate the performance of the proposed model. Visual Studio 2010 was employed to communicate with VISSIM to control and change the signal plan via Component Object Model interface (VISSIM-COM module).

Before simulation, some parameters (or models) in VISSIM need to be determined first so that to ensure that the simulation is closer to a real traffic system. According to Righol's report as well as Ding's study (Ding et al. 2015), Wiedemann 99 car-following model was suitable for urban traffic simulation in China. In this paper, Wiedemann 99 with the recommended values from Righol's report (i.e. $3.45 \mathrm{~m}$ for the car-following variance CC2 and $4.5 \mathrm{~m}$ - for car-following threshold CC3) was adopted.

To sufficiently populate the road with traffic, simulation time was set to 75 minutes, with the first 15 minutes being 'seeding time'. Since the VISSIM is stochas- tic model, some small differences in results are to be expected with different random seeds. To address this issue, each simulation was run 5 times with different random seeds. The reported simulation results, then, are the average over 5 runs.

In the simulation, some key parameters were varied to test the impact on the proposed control model, as follows:

- traffic demand: five volume-to-capacity ratios at $0.54,0.62,0.69,0.74$ and $0.81(70,80,90,100$ and $105 \%$ of the basic flow rate in Table 3) were test;

- headway: the average headways of BRT buses were set to $622,502,382$ and $262 \mathrm{~s}$ for both directions (these four headways were chosen because that the first bus can arrive at the intersection in different phases with different four headways; besides, the least common multiple of the headway and the cycle length is at least 3.6 hours, that made it possible for buses to arrive at the intersection in different phases during one simulation);

- maximum allowable saturation degree: $x_{i}^{\max }$ increased from 0.9 to 1 , with an interval of 0.01 .

Additionally, to evaluate the improvement of the proposed model, another two control strategies were employed to make a comparison: Control a: fixed-time control without TSP, and Control b: the conventional TSP control. To ensure a fair comparison, the background signal timing plans for these three control models were set to the same.

\section{Result Analysis}

In this section, the simulation results of the proposed TSP strategy, including delay time, stop rate, number of compressed phases, and the influence of saturation degree on TSP performance, were analysed and discussed further.

\subsection{Delay Reduction Performance for Buses and Other Vehicles}

The major objective of TSP is to reduce the delay for buses. Furthermore, the implementation of TSP should not result in a significant delay increase for other vehicles. Table 4 demonstrates the performance of the proposed TSP control model as well as other two models. Two main findings can be summarized from the table, as follows:

- the proposed model outperforms the fixed-time control and the conventional TSP control under all traffic demand scenarios in terms of bus delay. When volume-to-capacity ratios are set to 0.54 , $0.62,0.69,0.77$ and 0.81 , the average bus delays caused by proposed model are 3.23, 4.14, 7.9, 9.6 and $10.83 \mathrm{~s}$ respectively, and average bus delays caused by the conventional TSP control model are $8.81,10.78,11.9,13.3$ and 13.7 s. Compared with the conventional TSP control model, the proposed model can reduce the average bus delay by $63.37,61.63,33.37,27.55$ and $20.93 \%$, respec- 
tively. The improvement of proposed model over the fixed-time control is even better;

- the proposed model will not result in a large increase of average delay for other vehicles, sometimes it can even reduce the delay. When $v / c$ ratios are set to 0.69 and 0.81 , the increases in average delay for other vehicles are only 0.53 and $1.8 \%$ when compared with the fixed-time control, and 2.29 and $1.46 \%$ when compared with the conventional TSP control. The delay increase for other vehicles caused by the proposed model is very slight, which indicates the new TSP control model can handle the bus priority well while also ensuring the traffic efficiency for other vehicles. Note that, the average delay for other vehicles is reduced with low traffic demand. This is because the proposed model will select and compress the phases with the lowest volumes so that fewer vehicles will be affected by TSP.

\subsection{Other Performance Improvements Analysis}

Except for the reduction on bus delay, there are also other performance improvements. Fig. 4 illustrates a stop rate comparison under different traffic demands and headways. In all headway and demand scenarios, a proposed model performs better than the conventional TSP control model: the percentage of buses passing through the intersection without a stop is higher when a proposed model is employed. Especially, when the traffic demand is lower than 0.62 , the proposed TSP control model can ensure that all the buses can go across the intersection with no stopped delay, while the conventional TSP control model can only let around $60 \%$ of buses to proceed unimpeded through the intersection. Note that when headway and $v / c$ ratio are set to $502 \mathrm{~s}$ and 0.62 , the percentage of buses passing through the intersection without a stop are $100 \%$ with proposed TSP control model, and $0 \%$ with the conventional TSP control model. This demonstrates that compressing multi-

Table 4. Comparison results of model performance

\begin{tabular}{|c|c|c|c|c|c|c|}
\hline \multirow{2}{*}{$\begin{array}{l}\text { Intersection } \\
\text { approaches }\end{array}$} & \multirow{2}{*}{$\begin{array}{c}\text { Traffic } \\
\text { demand } v / c\end{array}$} & \multicolumn{3}{|c|}{ Average delay [s/veh] } & \multirow{2}{*}{$\begin{array}{l}\text { Improvement over } \\
\text { Control a [\%] }\end{array}$} & \multirow{2}{*}{$\begin{array}{c}\text { Improvement over } \\
\text { Control b [\%] }\end{array}$} \\
\hline & & Control a & Control b & $\begin{array}{c}\text { Proposed } \\
\text { control }\end{array}$ & & \\
\hline EB & \multirow{6}{*}{0.54} & 31.76 & 32.02 & 32.36 & -1.91 & -1.07 \\
\hline NB & & 29.19 & 29.08 & 28.56 & 2.16 & 1.80 \\
\hline WB & & 30.24 & 31.32 & 30.97 & -2.44 & 1.09 \\
\hline SB & & 28.82 & 29.04 & 28.24 & 2.01 & 2.76 \\
\hline Average & & 29.53 & 29.75 & 29.27 & 0.90 & 1.64 \\
\hline NB\&SB (BUS) & & 25.02 & 8.81 & 3.23 & 87.11 & 63.37 \\
\hline EB & \multirow{6}{*}{0.62} & 32.16 & 32.44 & 33.12 & -2.99 & -2.07 \\
\hline NB & & 30.01 & 30.23 & 29.34 & 2.23 & 2.96 \\
\hline WB & & 30.94 & 31.28 & 31.02 & -0.24 & 0.86 \\
\hline SB & & 28.65 & 28.72 & 28.74 & -0.31 & -0.07 \\
\hline Average & & 29.89 & 30.07 & 29.84 & 0.16 & 0.77 \\
\hline NB\&SB (BUS) & & 25.02 & 10.78 & 4.14 & 83.46 & 61.63 \\
\hline EB & \multirow{6}{*}{0.69} & 32.7 & 33.1 & 33.1 & -1.12 & 0.01 \\
\hline NB & & 31.6 & 31.1 & 31.4 & 0.86 & -0.86 \\
\hline WB & & 30.5 & 30.5 & 31.3 & -2.66 & -2.70 \\
\hline SB & & 30.9 & 29.9 & 31.2 & -0.86 & -4.33 \\
\hline Average & & 31.3 & 30.8 & 31.5 & -0.53 & -2.29 \\
\hline NB\&SB (BUS) & & 25.0 & 11.9 & 7.9 & 68.45 & 33.37 \\
\hline EB & \multirow{6}{*}{0.77} & 33.3 & 33.3 & 33.2 & 0.51 & 0.32 \\
\hline NB & & 31.7 & 31.8 & 31.7 & -0.01 & 0.18 \\
\hline WB & & 30.6 & 30.8 & 30.6 & -0.04 & 0.44 \\
\hline SB & & 32.6 & 33.1 & 31.5 & 3.26 & 4.61 \\
\hline Average & & 32.2 & 32.4 & 31.7 & 1.38 & 2.03 \\
\hline NB\&SB (BUS) & & 25.0 & 13.3 & 9.6 & 61.62 & 27.55 \\
\hline $\mathrm{EB}$ & \multirow{6}{*}{0.81} & 32.58 & 33.10 & 33.55 & -2.96 & -1.34 \\
\hline NB & & 31.81 & 31.80 & 32.15 & -1.06 & -1.09 \\
\hline WB & & 29.75 & 29.97 & 31.21 & -4.91 & -4.15 \\
\hline SB & & 31.52 & 31.54 & 31.86 & -1.08 & -1.01 \\
\hline Average & & 31.55 & 31.65 & 32.11 & -1.80 & -1.46 \\
\hline NB\&SB (BUS) & & 25.02 & 13.70 & 10.83 & 56.71 & 20.92 \\
\hline
\end{tabular}


a)

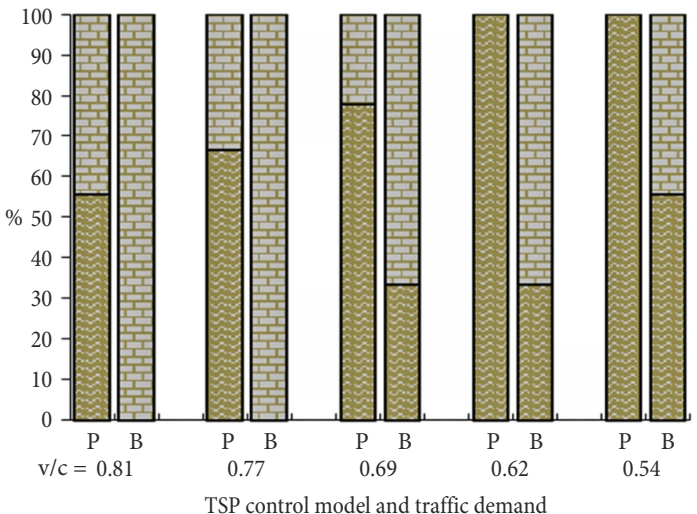

c)

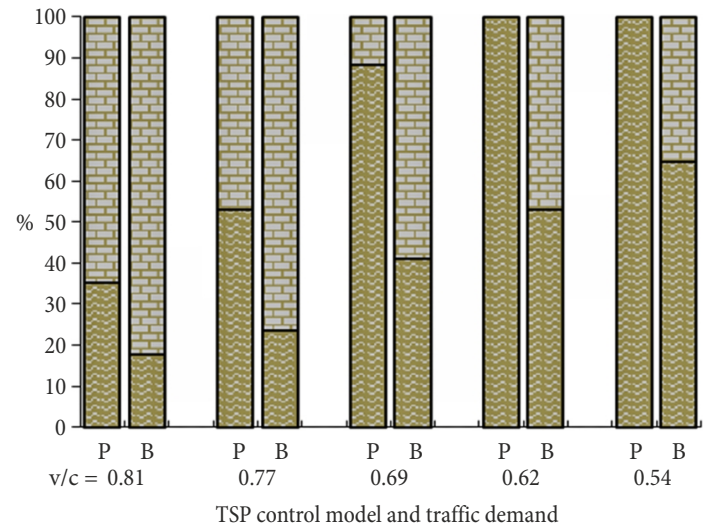

b)

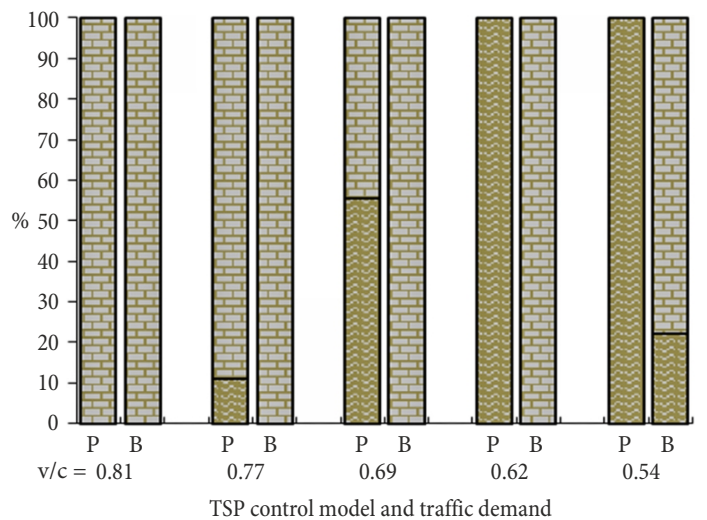

d)

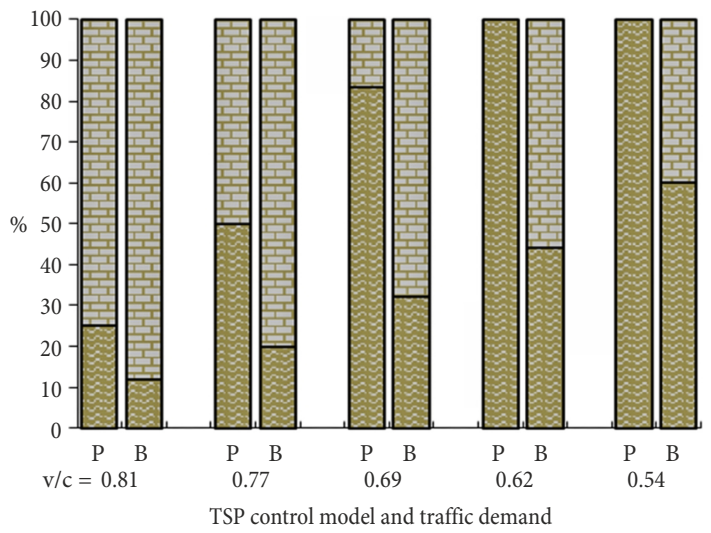

Buses get though intersection with stop $\quad$ Buses get though intersection without stop

Fig. 4. Stop rate comparison (note: $\mathrm{P}-$ Proposed TSP control model; $\mathrm{B}-$ Control b): $\mathrm{a}-$ headway $=622 \mathrm{~s}$; $\mathrm{b}-$ headway $=502 \mathrm{~s} ; \mathrm{c}-$ headway $=382 \mathrm{~s} ; \mathrm{d}-$ headway $=262 \mathrm{~s}$

phases can reduce the stop rate for buses to improve the transit efficiency.

Table 5 illustrates the number of compressed phases by the proposed TSP model. The average number of compressed phases ranges from 2.3 to 3.2. A significant increase trend of number of compressed phases can be observed when traffic demand increases. It makes sense because when the traffic demand is high, each phase will have less compressible time for buses. Under this situation, more phases are compressed together to provide time for a TSP request. Conversely, when the traffic demand decreases, there will be more compressible time for each phase, thus leading to the reduction of compressed phase number.

\subsection{Impact of Maximum Saturation Degree on Control Performance}

To better understand the performance of the proposed model under different maximum allowable saturation degrees, a further investigation was made to explore the relationship between maximum allowable saturation degree and average delay.

Table 6 illustrates the impact of maximum saturation degree on control performance. When traffic demand is low (Table $6, v / c=0.54$ ), the impact of maximum saturation degree on average delay of both control models are small. With the increase of maximum satura-
Table 5. Number of phases compressed for bus priority request

\begin{tabular}{|c|c|c|c|c|c|c|}
\hline \multirow[t]{2}{*}{$\begin{array}{c}\text { Headway } \\
{[\mathrm{s}]}\end{array}$} & \multirow{2}{*}{$\begin{array}{l}\text { Travel } \\
\text { demand } \\
v / c\end{array}$} & \multicolumn{4}{|c|}{$\begin{array}{c}\text { Percentage of a specified } \\
\text { number of phases } \\
\text { compressed [\%] }\end{array}$} & \multirow{2}{*}{$\begin{array}{l}\text { Average } \\
\text { No of } \\
\text { compressed } \\
\text { phases }\end{array}$} \\
\hline & & 1 & 2 & 3 & 4 & \\
\hline \multirow{5}{*}{622} & 0.81 & 0.0 & 0.0 & 77.8 & 22.2 & 3.2 \\
\hline & 0.77 & 0.0 & 11.1 & 77.8 & 11.1 & 3.0 \\
\hline & 0.69 & 0.0 & 44.4 & 44.4 & 11.1 & 2.7 \\
\hline & 0.62 & 0.0 & 77.8 & 11.1 & 11.1 & 2.3 \\
\hline & 0.54 & 11.1 & 55.6 & 22.2 & 11.1 & 2.3 \\
\hline \multirow{5}{*}{502} & 0.81 & 0.0 & 0.0 & 100.0 & 0.0 & 3.0 \\
\hline & 0.77 & 0.0 & 0.0 & 100.0 & 0.0 & 3.0 \\
\hline & 0.69 & 0.0 & 22.2 & 77.8 & 0.0 & 2.8 \\
\hline & 0.62 & 0.0 & 55.6 & 44.4 & 0.0 & 2.4 \\
\hline & 0.54 & 0.0 & 33.3 & 33.3 & 33.3 & 3.0 \\
\hline \multirow{5}{*}{382} & 0.81 & 0.0 & 7.1 & 69.4 & 23.5 & 3.2 \\
\hline & 0.77 & 11.8 & 29.4 & 41.2 & 17.6 & 2.6 \\
\hline & 0.69 & 17.6 & 41.2 & 41.2 & 0.0 & 2.2 \\
\hline & 0.62 & 7.1 & 57.6 & 24.7 & 10.6 & 2.4 \\
\hline & 0.54 & 18.8 & 58.8 & 10.6 & 11.8 & 2.2 \\
\hline \multirow{5}{*}{262} & 0.81 & 0.0 & 8.3 & 70.8 & 20.8 & 3.1 \\
\hline & 0.77 & 8.3 & 20.8 & 54.2 & 16.7 & 2.8 \\
\hline & 0.69 & 12.5 & 41.7 & 33.3 & 12.5 & 2.5 \\
\hline & 0.62 & 12.5 & 58.3 & 20.8 & 8.3 & 2.3 \\
\hline & 0.54 & 13.0 & 47.8 & 17.4 & 21.7 & 2.5 \\
\hline
\end{tabular}


tion degree, the bus delay time will decrease while the compression time per cycle will increase. Additionally, the other vehicles' average delay will not change a lot. That is because when the traffic volume is low, compressing a little green time will not significantly affect the running of other vehicles. Besides, the compression time by proposed TSP control model is double the number by control $b$, but the average bus delay by proposed model is $50 \%$ less than the number by Control B. These findings indicate that the proposed TSP control model is more efficient to provide green time for priority requests.

When traffic demand is high, as shown in Table 6 $(v / c=0.81)$, the results are somewhat different. When the maximum saturation degree is smaller than 0.93 , phase time cannot be compressed by both control models. That is mainly because the priority windows are too short to afford the time needed. When the maximum saturation degree is set to 0.94 , some phases are compressed and the bus delay reduction emerges by the proposed model. While the conventional TSP model cannot give signal priority to buses until the maximum saturation degree increases to 0.96 . All in all, the proposed TSP control model outperforms the conventional TSP model under all maximum saturation degree scenarios in terms of bus delay reduction. When the traffic demand is high, the proposed TSP control works earlier to serve TSP requests.

\section{Conclusions}

This study presents a TSP control model for isolated intersections. The proposed model aims to minimize bus delay by optimizing signal control phase selection and compression process, and meanwhile to reduce the impact of TSP on other vehicles. In the proposed model, the time that a TSP request needs is provided by compressing phase(s) with the lowest volume, and by multiphases if one single phase cannot afford the time needed. Field data were collected from a major corridor in Changzhou (China) and applied for VISSIM simulation. To evaluate the performance of the proposed model, the conventional TSP control and fixed-time control models were introduced. The comparative results showed that the proposed model performed the best, which reduced the average bus delay by at least $20.92 \%$ when compared with the conventional TSP control model (the maximum saturation degree was equal to 1). In addition, the impact of TSP implementation on other vehicles by proposed model was very slight: the largest increase of other vehicles delay was $2.29 \%$. Besides, the proposed model can also reduce the stop rate for buses.

The contribution of the paper is the two changes in how to select and compress signal control phases for a TSP request. First, the time that a TSP request needs

Table 6. Impact of maximum saturation degree on control performance

\begin{tabular}{|c|c|c|c|c|c|c|c|c|}
\hline \multirow{3}{*}{$\begin{array}{c}\text { Maximum } \\
\text { saturation } \\
\text { degree }\end{array}$} & \multicolumn{3}{|c|}{ Control b } & \multicolumn{3}{|c|}{ Proposed Control } & \multirow{2}{*}{\multicolumn{2}{|c|}{$\begin{array}{l}\text { Improvement ove } \\
\text { Control b [\%] }\end{array}$}} \\
\hline & \multirow{2}{*}{$\begin{array}{l}\text { Compression } \\
\text { time }[\mathrm{s} / \text { cycle }]\end{array}$} & \multicolumn{2}{|c|}{ Average delay [s/veh] } & \multirow{2}{*}{$\begin{array}{l}\text { Compression } \\
\text { time [s/cycle] }\end{array}$} & \multicolumn{2}{|c|}{ Average delay [s/veh] } & & \\
\hline & & other & bus & & other & bus & other & bus \\
\hline \multicolumn{9}{|c|}{$v / c=0.54$} \\
\hline 0.90 & 1.27 & 29.78 & 10.54 & 2.49 & 29.83 & 4.15 & -0.18 & 60.62 \\
\hline 0.91 & 1.27 & 29.78 & 10.54 & 2.73 & 29.48 & 3.37 & 0.99 & 68.01 \\
\hline 0.92 & 1.31 & 29.78 & 10.54 & 2.73 & 29.44 & 3.37 & 1.13 & 68.01 \\
\hline 0.93 & 1.31 & 29.78 & 10.54 & 2.73 & 29.44 & 3.37 & 1.13 & 68.01 \\
\hline 0.94 & 1.31 & 29.78 & 10.54 & 2.73 & 29.44 & 3.37 & 1.13 & 68.01 \\
\hline 0.95 & 1.38 & 29.81 & 10.24 & 2.73 & 29.77 & 3.37 & 0.15 & 67.06 \\
\hline 0.96 & 1.38 & 29.81 & 10.24 & 2.73 & 29.77 & 3.37 & 0.15 & 67.06 \\
\hline 0.97 & 1.40 & 29.84 & 8.92 & 2.79 & 29.63 & 3.28 & 0.73 & 63.29 \\
\hline 0.98 & 1.40 & 29.84 & 8.92 & 2.79 & 29.63 & 3.28 & 0.73 & 63.29 \\
\hline 0.99 & 1.40 & 29.84 & 8.92 & 2.79 & 29.63 & 3.28 & 0.73 & 63.29 \\
\hline 1.00 & 1.40 & 29.75 & 8.81 & 2.79 & 29.27 & 3.23 & 0.73 & 63.29 \\
\hline \multicolumn{9}{|c|}{$v / c=0.81$} \\
\hline 0.90 & 0.00 & 31.55 & 25.02 & 0.00 & 31.55 & 25.02 & 0.00 & 0.00 \\
\hline 0.91 & 0.00 & 31.55 & 25.02 & 0.00 & 31.55 & 25.02 & 0.00 & 0.00 \\
\hline 0.92 & 0.00 & 31.55 & 25.02 & 0.00 & 31.55 & 25.02 & 0.00 & 0.00 \\
\hline 0.93 & 0.00 & 31.55 & 25.02 & 0.00 & 31.55 & 25.02 & 0.00 & 0.00 \\
\hline 0.94 & 0.00 & 31.55 & 25.02 & 0.09 & 31.70 & 24.69 & 4.68 & 1.57 \\
\hline 0.95 & 0.00 & 31.55 & 25.02 & 0.13 & 32.30 & 24.46 & 2.87 & 2.46 \\
\hline 0.96 & 0.04 & 31.85 & 24.89 & 0.18 & 31.34 & 24.23 & 1.58 & 2.65 \\
\hline 0.97 & 0.04 & 31.85 & 24.89 & 0.18 & 31.34 & 24.23 & 1.58 & 2.65 \\
\hline 0.98 & 0.04 & 31.85 & 24.89 & 1.00 & 30.93 & 18.40 & 2.89 & 26.08 \\
\hline 0.99 & 0.09 & 32.06 & 24.69 & 1.04 & 31.87 & 18.20 & 0.59 & 26.26 \\
\hline 1.00 & 0.09 & 31.65 & 13.70 & 1.20 & 32.11 & 10.83 & -1.46 & 20.90 \\
\hline
\end{tabular}


is not provided by the nearby phase(s) any more, but by the phase(s) with the lowest traffic volume; second, multi-phases can be selected to serve the bus request together. These two changes make the proposed TSP control model a bit more complicated, but the performance is quite well under all scenarios.

Note that the good performance of the proposed model is mainly based on the accurate bus arrival time prediction result. In the proposed model, bus arrival time should be predicted one cycle length ahead to ensure that the model can have enough time to adjust starting and ending time of all phases. Actually, to predict the bus arrival time one cycle length ahead is difficult. This is the main limitation to implement the proposed model. Further extension of this paper as well as the implementation of the proposed model should pay more attention on this aspect.

\section{Acknowledgements}

This study is supported by National Key Basic Research Program of China (No. 2012CB725402), National Hightech R\&D Program of China (No SS2014AA110303) and Scientific Research Foundation of Graduate School of Southeast University. The authors also appreciate the STAR Lab of the University of Washington for the help and technical support.

\section{References}

Ahmed, B. 2014. Exploring new bus priority methods at isolated vehicle actuated junctions, Transportation Research Procedia 4: 391-406. https://doi.org/10.1016/j.trpro.2014.11.030

Al-Sahili, K.; Taylor, W. 1996. Evaluation of bus priority signal strategies in Ann Arbor, Michigan, Transportation Research Record: Journal of the Transportation Research Board 1554: 74-79. https://doi.org/10.3141/1554-10

Balke, K.; Dudek, C.; Urbanik, T. 2000. Development and evaluation of intelligent bus priority concept, Transportation Research Record: Journal of the Transportation Research Board 1727: 12-19. https://doi.org/10.3141/1727-02

Chang, E. C.-P.; Messer, C. J. 1985. Minimum Delay Optimization of a Maximum Bandwidth Solution to Arterial Signal Timing (Abridgment), Transportation Research Record 1005: 89-95.

Christofa, E.; Skabardonis, A. 2011. Traffic signal optimization with application of transit signal priority to an isolated intersection, Transportation Research Record: Journal of the Transportation Research Board 2259: 192-201. https://doi.org/10.3141/2259-18

Delgado, F.; Muñoz, J. C.; Giesen, R.; Wilson, N. H. M. 2015. Integrated real-time transit signal priority control for highfrequency segregated transit services, Transportation Research Record: Journal of the Transportation Research Board 2533: 28-38. https://doi.org/10.3141/2533-04

Ding, J.; Yang, M.; Wang, W.; Xu, C.; Bao, Y. 2015. Strategy for multiobjective transit signal priority with prediction of bus dwell time at stops, Transportation Research Record: Journal of the Transportation Research Board 2488: 10-19.

https://doi.org/10.3141/2488-02
Dion, F.; Hellinga, B. 2002. A rule-based real-time traffic responsive signal control system with transit priority: application to an isolated intersection, Transportation Research Part B: Methodological 36(4): 325-343.

https://doi.org/10.1016/S0191-2615(01)00006-6

Duerr, P. 2000. Dynamic right-of-way for transit vehicles: integrated modeling approach for optimizing signal control on mixed traffic arterials, Transportation Research Record: Journal of the Transportation Research Board 1731: 31-39. https://doi.org/10.3141/1731-05

Ekeila, W.; Sayed, T.; El Esawey, M. 2009. Development of dynamic transit signal priority strategy, Transportation Research Record: Journal of the Transportation Research Board 2111: 1-9. https://doi.org/10.3141/2111-01

Garrow, M.; Machemehl, R. 1997. Development and Evaluation of Transit Signal Priority Strategies, Research Report SWUTC/97/472840-00068-1. Southwest Region University Transportation Center, Center for Transportation Research, University of Texas at Austin, Austin, Texas, US. 148 p.

Hu, J.; Park, B. B.; Lee, Y.-J. 2015. Coordinated transit signal priority supporting transit progression under connected vehicle technology, Transportation Research Part C: Emerging Technologies 55: 393-408.

https://doi.org/10.1016/j.trc.2014.12.005

Hu, J.; Park, B.; Parkany, A. 2014. Transit signal priority with connected vehicle technology, Transportation Research Record: Journal of the Transportation Research Board 2418: 20-29. https://doi.org/10.3141/2418-03

Jacobson, J.; Sheffi, Y. 1981. Analytical model of traffic delays under bus signal preemption: theory and application, Transportation Research Part B: Methodological 15(2): 127138. https://doi.org/10.1016/0191-2615(81)90039-4

Janos, M.; Furth, P. 2002. Bus priority with highly interruptible traffic signal control: simulation of San Juan's Avenida Ponce de Leon, Transportation Research Record: Journal of the Transportation Research Board 1811: 157-165. https://doi.org/10.3141/1811-19

Khasnabis, S.; Reddy, G. V.; Chaudry, B. B. 1991. Signal preemption as a priority treatment tool for transit demand management, in Vehicle Navigation and Information Systems Conference, 20-23 October 1991, Troy, MI, USA, 2: 1093-1104. https://doi.org/10.1109/VNIS.1991.205855

Kim, W.; Rilett, L. 2005. Improved transit signal priority system for networks with nearside bus stops, Transportation Research Record: Journal of the Transportation Research Board 1925: 205-214. https://doi.org/10.3141/1925-21

Lee, J.; Shalaby, A.; Greenough, J.; Bowie, M.; Hung, S. 2005. Advanced transit signal priority control with online microsimulation-based transit prediction model, Transportation Research Record: Journal of the Transportation Research Board 1925: 185-194. https://doi.org/10.3141/1925-19

Li, J.; Wang, W.; Van Zuylen, H.; Sze, N.; Chen, X.; Wang, H. 2012. Predictive strategy for transit signal priority at fixedtime signalized intersections: case study in Nanjing, China, Transportation Research Record: Journal of the Transportation Research Board 2311: 124-131. https://doi.org/10.3141/2311-12

Li, R.; Zhang, X. 2012. Bus rapid transit signal priority strategy based on schedule, in Transportation Research Board 91st Annual Meeting, 22-26 January 2012, Washington, DC, US, $1-16$. 
Liao, C.-F.; Davis, G. 2007. Simulation study of bus signal priority strategy: taking advantage of global positioning system, automated vehicle location system, and wireless communications, Transportation Research Record: Journal of the Transportation Research Board 2034: 82-91. https://doi.org/10.3141/2034-10

Lin, W.-H. 2002. Quantifying delay reduction to buses with signal priority treatment in mixed-mode operation, Transportation Research Record: Journal of the Transportation Research Board 1811: 100-106.

https://doi.org/10.3141/1811-12

Ma, W.; Liu, Y.; Yang, X. 2013. A dynamic programming approach for optimal signal priority control upon multiple high-frequency bus requests, Journal of Intelligent Transportation Systems: Technology, Planning, and Operations 17(4): 282-293.

https://doi.org/10.1080/15472450.2012.729380

Ma, W.; Yang, X. 2007. A passive transit signal priority approach for bus rapid transit system, in IEEE Intelligent Transportation Systems Conference, 2007: ITSC 2007, 30 September - 3 October 2007, Seattle, WA, US, 413-418. https://doi.org/10.1109/ITSC.2007.4357625

Polgár, J.; Tettamanti, T.; Varga, I. 2013. Passenger number dependent traffic control in signalized intersections, Periodica Polytechnica Civil Engineering 57(2): 201-210. https://doi.org/10.3311/PPci.7175

Salter, R. J.; Shahi, J. 1979. Prediction of effects of bus-priority schemes by using computer simulation techniques, Transportation Research Record 718: 1-5.

Smith, H. R.; Hemily B.; Ivanovic, M. 2005. Transit Signal Priority (TSP): a Planning and Implementation Handbook. ITS America. 200 p.

Tan, C.-W.; Park, S.; Liu, H.; Xu, Q.; Lau, P. 2008. Prediction of transit vehicle arrival time for signal priority control: algorithm and performance, IEEE Transactions on Intelligent Transportation Systems 9(4): 668-696.

https://doi.org/10.1109/TITS.2008.2006799

US Department of Housing and Urban Development. 1968a. Study of Evolutionary Urban Transportation. Vol. 1, Washington, DC, US.

US Department of Housing and Urban Development. 1968b. Study of Evolutionary Urban Transportation. Vol. 2, Washington, DC, US.

US Department of Housing and Urban Development. 1968c. Study of Evolutionary Urban Transportation. Vol. 3, Washington, DC, US.

Wahlstedt, J. 2011. Impacts of bus priority in coordinated traffic signals, Procedia - Social and Behavioral Sciences 16: 578-587. https://doi.org/10.1016/j.sbspro.2011.04.478

Wang, Y.; Ma, W.; Yin, W.; Yang, X. 2014. Implementation and testing of cooperative bus priority system in connected vehicle environment: case study in Taicang City, China, Transportation Research Record: Journal of the Transportation Research Board 2424: 48-57. https://doi.org/10.3141/2424-06

Wolput, B.; Tegenbos, R.; Skabardonis, A.; Tampère, C. M. 2015. Control strategies for transit priority: comparing adaptive priority with full transit signal priority, in Transportation Research Board 94th Annual Meeting, 11-15 January 2015, Washington, DC, US, 1-15.

Zeng, X.; Zhang, Y.; Balke, K. N.; Yin, K. 2014. A real-time transit signal priority control model considering stochastic bus arrival time, IEEE Transactions on Intelligent Transportation Systems 15(4): 1657-1666.

https://doi.org/10.1109/TITS.2014.2304516
Zhou, G.; Gan, A. 2009. Design of transit signal priority at signalized intersections with queue jumper lanes, Journal of Public Transportation 12(4): 117-132.

https://doi.org/10.5038/2375-0901.12.4.7

\section{APPENDIX}

The implementation of the proposed TSP strategy is as simple as previous real-time TSP strategies (Balke et al. 2000; Duerr 2000; Christofa, Skabardonis 2011). The framework of the proposed TSP system is shown in Fig. A1. The proposed TSP system mainly consists of three components: Signal Control Unit (SCU), On-Bus Unit (OBU) and Control Process Unit (CPU).

SCU can be installed near intersections, which is composed of signal control facilities (e.g., signal lights) and detectors. The detectors should be equipped on each lane to collect traffic arrival data by direction and by lane in real time. OBU is installed on buses to collect and provide real-time bus location and speed information. OBU mainly contains Automatic Vehicle Location (AVL) system and On-Board Diagnostic (OBD). CPU is the brain of the TSP system, which deals with all the information received from SCU and OBU and then determines the status of the signal light according to the proposed TSP strategy. CPU is consisted of a built-in history database, and a computer with proposed TSP strategy inside. The information transfer between CPU and SCU/OBU is via wireless communication technologies, such as $4 \mathrm{G}$ and Dedicated Short-Range Communications (DSRC).

Two issues in Fig. A1 need to pay more attentions. One is how to measure the input parameters; another one is how to apply the proposed TSP strategy.

The data required for the input parameters measurement are quite simple, which are similar to previous studies. Two kinds of data are necessary: history data, which are pre-collected and stored in the CPU, and realtime data, which are measured by SCU and OBU in real time. The history data are the same as those in previous studies. The real-time data, including traffic arrival and GPS-based bus data, are the key information for input parameters measurement. The traffic arrival information contains traffic flow rates and traffic components of each lane. These can be measured by traditional traffic detectors, e.g., loop detectors. In order to get the arrival data of every lane, each lane should be equipped with one separate detector. GPS-based bus information are collected by AVL system and transmitted to CPU automatically.

As for TSP strategy application issue, the proposed TSP strategy is still a kind of TSP algorithm, so it is easy to implement the proposed TSP strategy following the framework in Fig. A1, or from an existing TSP system. For an existing real-time TSP system, the newly proposed TSP can be implemented by replacing the original TSP strategy. Besides, the proposed TSP strategy can be easily updated or replaced as well in the possible future. 


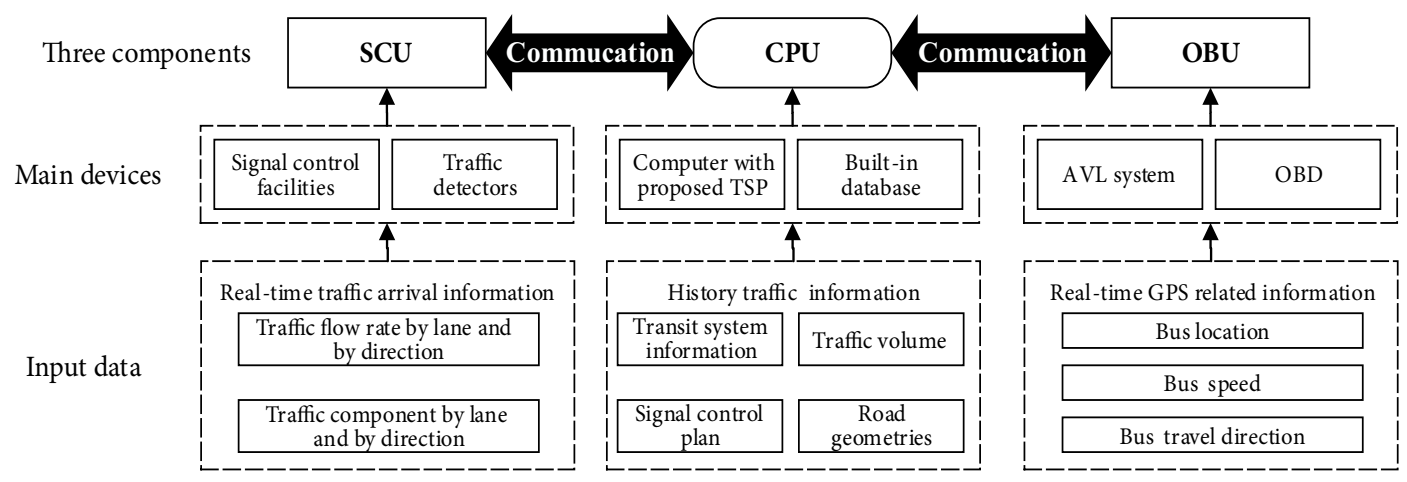

Fig. A1. The framework of the proposed TSP implementation 